\title{
InfoPlant: Multimodal augmentation of plants for enhanced human-computer interaction
}

\author{
Jan Hammerschmidt*, Thomas Hermann*, Alex Walender, Niels Krömker \\ *Ambient Intelligence Group \\ Bielefeld University, Germany \\ E-mail: \{jhammers,thermann,awalende,nkroemke\}@techfak.uni-bielefeld.de
}

\begin{abstract}
In this work, we present and evaluate a novel ambient information display that is designed to provide unobtrusive yet engaging feedback. The basis of this display is a natural, living plant, which is augmented in several ways to enable it to indicate information in various different ways. We describe the design and the construction of the InfoPlant, discuss its different modalities and present two demonstrator systems, including a novel ecofeedback display. A subsequent study showed that the InfoPlant was indeed perceived as unobtrusive by the large majority of participants and that it was easily accepted as a possible new entity in a living-room context. Also, the provided feedback was assessed as generally very helpful and that it would make users aware of their resource consumption and could have an influence on their consumption behavior.
\end{abstract}

Keywords-Ambient information displays, Eco-Feedback, Physical computing, Sustainable HCI, Behavior Change

\section{INTRODUCTION}

A central part of Weiser's notion of Ubiquitous Computing is that of disappearing technology: devices that "weave themselves into the fabric of everyday life until they are indistiguishable from it" [1]. Derived from this vision, the concept of calm computing additionally emphasizes technology that explicitly engages the periphery of attention, meaning that it is able to offer information in a way that the user can attend to it without explicitly focussing on the respective device [2]. One way to work towards such "disappearing technology" is to explicitly design a new artifact to blend into its surroundings, which is typically done for ambient displays [3]. The proposed approach, however, would be to use an existing artifact that is well-established as an everyday object, or even appreciated as a beautiful addition to the environment, and technologically augment this artifact in order to enable it to function as an ambient display.

When thinking about possible entities with these qualities, several objects might come to mind, which are in some way important to the respective person. However, we think that few are as universally appreciated as plants are: Being part of our natural environment, they can be found almost everywhere, including in our homes and our offices. Furthermore, plants already have their own way of communicating to us some aspects of their overall state: Most of the time it's easy to tell if a plant needs more water, and for many plants we can see them turning towards a specific direction due to phototropism [4]. Finally, most people intuitively have a generally positive attitude towards plants, and, as "primal" natural entities in our surroundings, they are able to elicit feelings of connectedness and care, as has been hinted at in a recent study [5].

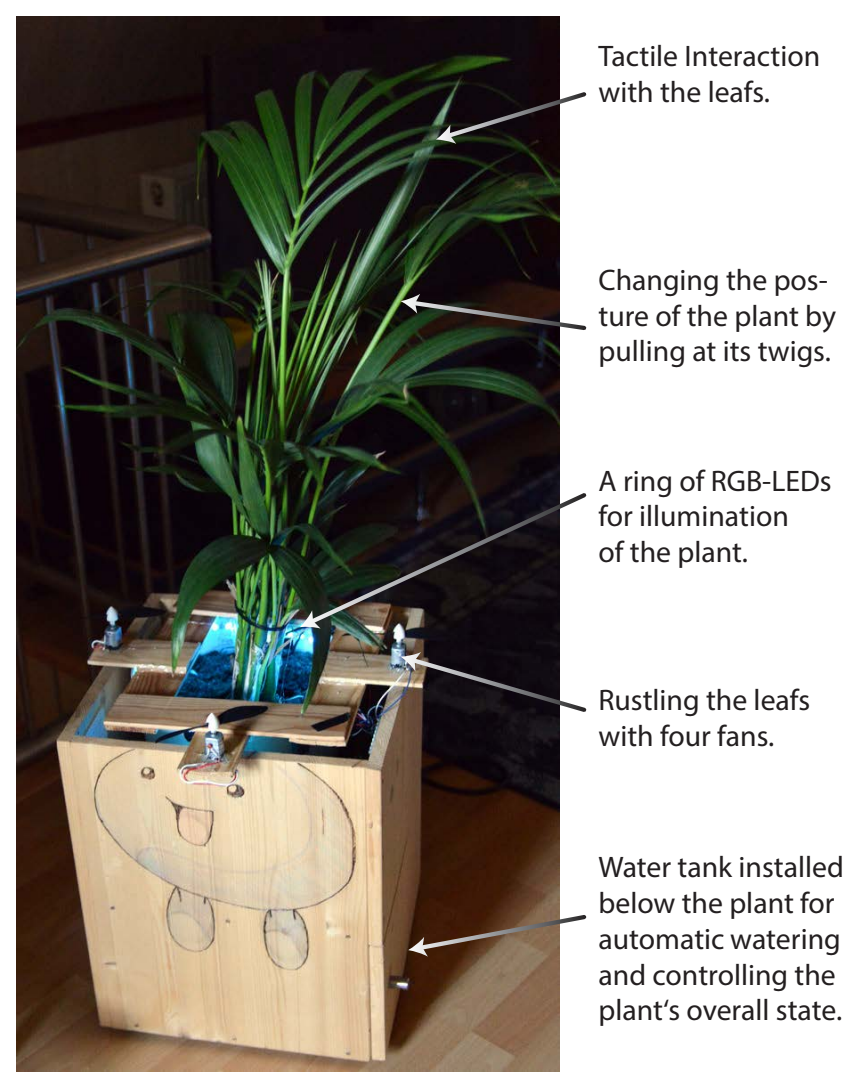

Fig. 1. Prototype of the InfoPlant, installed in a living environment.

This last aspect is of particular importance when thinking about using ambient information displays as eco-feedback devices [6]. Here, the presented information is supposed to lead to an adaptation of the user's consumption patterns, and ultimately to a decrease in overall expenditure. Due to aforementioned reasons, it is reasonable to assume that having an ambient display that is based on a plant will lead to an increased persuasiveness [7].

\section{RELATED WORK}

While there is a small body of work on using plants in a technological context and also as artifacts for interaction with humans, the aspect of unobtrusiveness and attention guiding as well as persuasiveness is rarely studied empirically.

The state of the art roughly falls into four categories, which will be surveyed in the following: 
Dealing with the broader topic of integrating plants into the technological world, Tanaka and Kuribayashi developed a toolkit for the design of artifacts for "human-plants-computer interaction" [8]. They propose two design strategies and several general patterns for plants being used in different ways [9]. On the sensory-side, the toolkit focuses on measuring so-called "biopotentials", which are somewhat difficult to analyze, but can give an indication of the general condition of the plant. As an example, the authors have developed a demonstrator system, where measurements of the plant's biopotentials are mapped onto the color and intensity of a range of LEDs installed within the flowerpot [10] (cp. Figure 2a). Changes in biopotential can occur due to the user watering the plant, changes in the environment, or the plant being touched by the user. In line with this example, the focus of their work lies less on the use of plants as ambient displays controlled by (external) data, but more on using the plant as a sensor. The authors also emphasize the notion of using the toolkit as a "versatile creative environment" for edutainment applications.

On a more abstract level, Goumopoulos et al. transform ordinary plants into "ePlants", which feature an additional layer for interfacing with the sorrounding (technological) environment [11]. Here, the focus lies primarily on the integration of plants into a larger distributed network in order to establish "mixed societies of communicating plants and artifacts", with both sensory and interactive communication and distributed decision-making. As a conceptual framework, the authors have developed an ontology that defines the possible relations between ePlants, sensors and eGadgets.

To the best of our knowledge, Holstius and colleagues are the first to use a plant in an eco-feedback context: They designed an "ambient plant display" that is effectively able to lean to the side and thereby can indicate the prevalence of one of typically two categories [5]. Using fast-growing corn seeds and two daylight bulbs, it was possible to change the direction of growth based on external input data and with this to produce a visible "lean" of the plant in a relatively short amount of time (cfp Figure 2b). Additionally, a robotic, biomimetric plant was developed, which emulates the looks of the natural one and is also able to tilt its leafs to the side. The authors evaluated and compared both designs in a university's cafeteria, giving feedback on the recycling behavior of its guests during a two-week field study: The largest increase in (relative) recycling could be observed for the natural plant display. Although the feasibility of this design depends on having a (fast-growing) plant in its growing-phase and only works with a considerable amount of lighting ${ }^{1}$, the conducted field study gives a first hint towards an enhanced efficacy of plants compared to artificial artifacts, which can, according to a number of short interviews, be attributed to feelings of appreciation and caring for the plant. Finally, the authors propose combining living and electromechanical components in a hybrid artifact.

Dealing more specifically with using a plant as interface technology, Poupyrev et al. are using the Swept Frequency Capacitive Sensing method developed by Sato et al. [12] to detect a user touching a plant's leafs [13]. For that they excite the plant with an electrical signal at several frequencies and

\footnotetext{
${ }^{1}$ According to the authors, a visible lean can be achieved with an exposure of $8 \mathrm{hrs} /$ day of a $100 \mathrm{~W}$ lightbulb in 3-4 days.
}

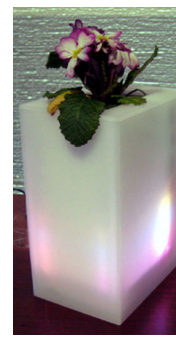

(a)

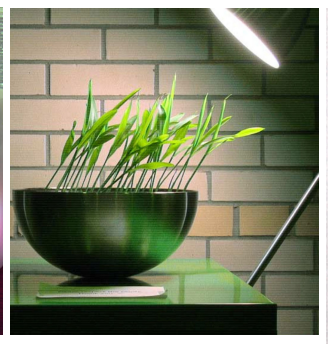

(b)

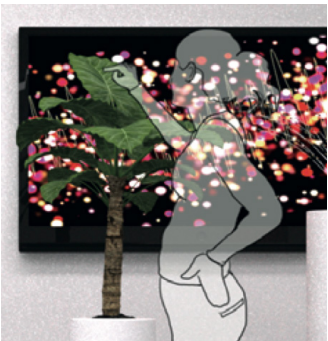

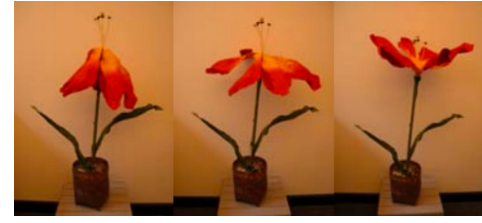

(c)

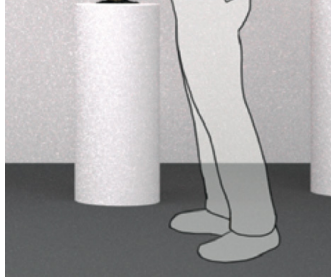

(d)
Fig. 2. Pictures of related work dealing with human-plant interaction (a) shows an augmented flowerpot with integrated LEDs indicating changes in the plant's biopotentials [10]. (b) depicts the controlling of a plant's growing direction through phototropism, used in [5]. (c) shows an electromechanically augmented artificial Lily that is able to let the blossom hang down or open it up to full bloom [14]. Finally, (d) illustrates a capacitive touch sensing method that can be used detect a user's interaction with the plant [13].

measure the response at each frequency to detect, if (and where) the user has touched the plant (Figure 2d).

Finally, moving away from living plants, Antifakos and Schiele augmented an artificial Lily (Figure 2c) with a mechanism to actuate the flower's petals, thereby being able to let the blossom hang down or open it up to full bloom [14]. Additionally equipped with a microphone, they used the flower as an unobtrusive ambient display in group meetings to show which participants are dominating the discussion - and which participants are left out of it. While no evaluation in terms of efficacy were conducted, the authors observed that the flower was very quickly accepted as part of the environment.

In summary, we can see that, while various ideas and application scenarios have been proposed, reseach in this direction is still only at the beginning. Also, no prototype has yet been developed to assess attention guiding capabilities of cues or long-term characteristics of plants used in a humancomputer interaction context.

\section{CONCEPT OF THE INFOPLANT}

fig:

An important aspect of an ambient display is the potential absorption of attention: As the display rarely presents information relevant for the primary activity of the user [15], a fundamental design goal is the unobtrusiveness of the system, meaning that in most cases, we would aim for a low attention absorption. However, having the potential to alert the user in order to (temporarily) bring the attention to a certain issue obviously adds to the versatility of the system, for example when dealing with so-called horizon activities, which are monitored with the intent to become the users's primary activity in the near future.

Another dimension that is particularly important for a natural ambient display like the InfoPlant is the possible speed 


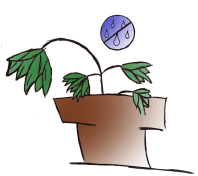

(1)

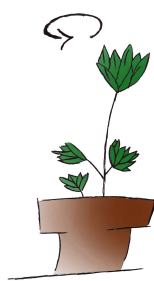

(2)

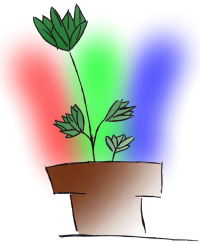

(3)

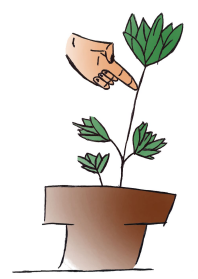

(4)

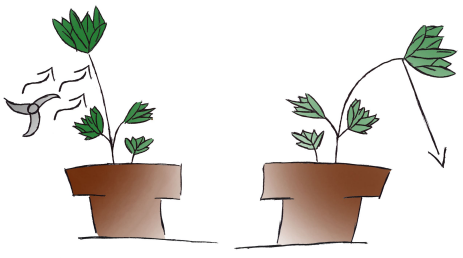

(5)

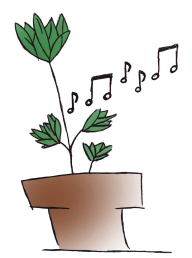

(7)

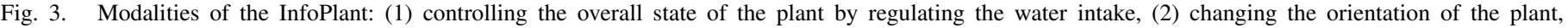

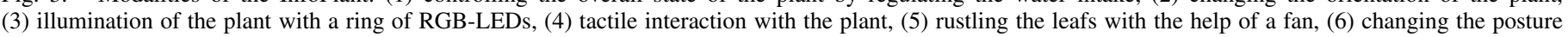
of the plant by tugging at a twig, and (7) integration of sound-ouput.

of change of the different modalities, as the plant itself can change its state only relatively slowly. In Figure 3, we can see several concept sketches of possible modalities - each with a different potential attention absorption and speed of change which will be discussed individually in the following.

1) Overall state of the plant: Although there are multiple ways of influencing the overall state of a plant (e.g. to mechanically control the posture of the plant, as in [14]), the most authentic change in appearance can be achieved by giving or withholding elementary elements for growth, which will then (indirectly) affect the plant's appearance [16]. While adjusting the intake of, for example, carbon and oxygen is largely unfeasible, as we would have to change the quality of the sorrounding air, there are three elements that are commonly used to influence (mostly improve) a plant's growth. These are (a) light, (b) water, and (c) mineral nutrients like nitrogen, phosphorus, and potassium. (a) Light is certainly a possibility to boost a plant's growth [17] (and can also change it's direction [5]). However, in order to also be able to negatively influence the appearance of the plant, we would have to fully control the lighting situation, i.e. completely prevent sunlight from reaching the plant, which is effectively impossible for most, if not all, situations. Similarly, the soil of the plant would have to be precisely monitored in order to (c) control the availability of nutrients for the plant, which is technically quite challenging. Also, not only would the visible changes in appearance be very slow, but also due to initial availability of nutrients in the soil, there would be no possibility of withholding them for a longer period of time. Finally, (b) watering the plant is comparatively easy to control, as we can both measure soil moisture and give (or withhold) water by employing a moisture sensor and a water pump. While the capacity to store water - both of the soil and the plant - makes it difficult to change this parameter quickly, for most cases, this is the fastest way of controlling the plant's appearance.

2) Orientation: A second way of influencing the overall appearance of the plant is by rotating it: In most cases, a plant can give the impression of "facing" the user - or, on the other hand, look away from them. Similar to the previous modality, this would change the plant's appearance on a "global" level. However, these changes could be made far quicker than by controlling the water intake of the plant and could be implemented by using a rotary plate beneath the flowerpot.
3) Illumination: Different from the previous modalities, augmenting the plant with artificial illumination would mean to definitely move from a purely natural artifact towards an (at least partially) artificial one. However, as discussed earlier, this cannot completely be avoided when aiming for an ambient display with a certain expressive power. Also, it should be possible to install a number of LEDs in way that they are barely to see directly, so that we can achieve a potentially quite unobtrusive passive lighting of the plant. After discussing a number of possibilities of how to integrate a single or multiple LEDs, we decided to install an RGB-LED-Stripe at the inside of the flowerpot, just above the soil, so that the plant can be illuminated from different sides and users can only see LEDs from the opposite side (provided that they look at the plant from the right angle and the plant itself is not obstructing them). All in all we gain a quite versatile modality for communicating with the user, both in terms of subtlety and in reaction time.

4) Tactile interaction: While primarily designed as an ambient display, which should be able to unobtrusively communicate information to the user, a certain level of interaction would allow for a much greater flexibility when developing applications for the InfoPlant and can be helpful for switching between different display modes or to actively query specific information. Based on work from Poupyrev et al. [13], we decided to integrate a capacitive touch-sensor into the InfoPlant, as it has the advantage of being relatively easy to install, it allows the user to actually get "in touch" with the plant, and it keeps the plant itself as natural as possible.

5) Leaf rustling: In the design process of the InfoPlant, we thought about which motions or movements of plants can also be observed for non-augmented species and therefore would be most natural for a "plant interface". Something that almost everyone knows is the wind rustling through the leafs of a plant, which generates a soft sound and a subtle motion. This event usually holds no other information than signalling the presence of wind. When generated for an indoor plant, however, this can be an additional unobtrusive modality for signalling an event or displaying a specific status. The most straightforward way of recreating this rustling of leafs is by using small fans to produce the needed amount of wind, which is what we also did for the InfoPlant.

6) Posture of the plant: Similar to controlling the overall state of the plant as dicussed in Section 1, actively modifying the posture, i.e. the position of branches and twigs can be used to mimic a natural change in appearance of the plant (also 
cp. [14]). Also, with a more precise control, additional ways of communication would be possible, like, for example, using a twig as a gauge for a continuous variable. Implementationwise, this probably is the most "intrusive" modality, as we have to get very near the plant to achieve the desired effect, and therefore also the most difficult one to realize in an unobtrusive way. Different to [14], who used an actual shaft to open and close petals of an (artificial) flower, we chose to attach nylon threads to the twigs of the plant, which are connected to small servos within the flowerpot. This way, the augmentation of the plant is still very subtle and only noticable when users are quite near of it.

7) Sound: Finally, the modality of sound opens up a completely new design space for the InfoPlant. It is easy to integrate, as we basically only need an additional loudspeaker, and the modality is extremely versatile insofar as it allows the use of a very broad range of sound designs. As a designer, one can draw on a large body of research on sonification and auditory displays [18] and make use of a range of methods from short sonic events to extended/stationary and even continuous sounds that display information. Also, sound can further accentuate and augment other events such as moving a twig or changes in illumination [19].

\section{CONSTRUCTION}

Construction of the InfoPlant can be divided into two phases: the prototyping phase and the integration phase. In the first phase, we used the $\mathrm{BRIX}_{2}$-platform [20] to freely experiment with the different modalities discussed in Section III. BRIX $_{2}$ is a framework for physical computing based on the Arduino project ${ }^{2}$, with small programmable, LEGO ${ }^{\circledR}$-compatible modules as its central components. Each module has several built-in sensors, a low-power radio transmitter for wireless communication between the individual modules, and additionally can be enhanced by a range of extension modules. For the prototyping phase, each modality was assigned its own BRIXmodule in order to allow for a more flexible and independent implementation. Rather soon in the process, we decided to concentrate on five modalities: (a) changing the overall state of the plant, (b) illumination, (c) tactile interaction, (d) leaf rustling, and (e) changing the plant's posture.

For (a) we used a commercially available soil moisture sensor [21] in combination with a low-cost industrial peristaltic pump and a small water tank. One advantage of using a peristaltic pump is the fact that it keeps the water in the connecting tubes instead of letting it flow back, which makes the water supply more precise. A major difficulty with this modality, however, is the question, when to give how much water in order to achieve a certain appearance of the plant. We discovered that the moisture level, where a plant not complely drying out, but still looks "sad" can be quite narrow, and we obviously want to prevent the plant to wither. Furthermore we have to deal with the fact that the control loop between water pump and moisture sensor has a quite large delay of several minutes, as the water accumulating in the flowerpot takes some time to be distributed in the soil. In order to have the most precise measuring of soil moisture, we collected the sensor's response to a range of moisture levels by putting a specific amount of water into several small containers filled

\footnotetext{
${ }^{2}$ http://www.arduino.cc, last accessed: 2015-07-02
}

with completely dried-out soil and logging the voltage readings of the sensor after waiting for 15 minutes. More difficult, however, is to define a direct translation from moisture level to the appearance of the plant, as it reacts to the given water with an even greater delay. While it was possible for us to define moisture thresholds for our setup, it seems to be difficult to find a set of universal parameters, which work for a broader range of plants.

For (b) the illumation of the plant, we used a strip of 24 RGBLEDs, which we installed on the inside of the flowerplot. This way, users normally do not see the LEDs directly but only the illuminated plant. In order to achieve a linear mapping between input values and brightness of the LEDs, we implemented a warping function to compensate for the nonlinearity of the LEDs. (c) Touch interaction was implemented using an Arduino-compatible variant [22] of the capacitive sensing method described in [12] and (d) leaf-rustling was achieved by installing 4 fans at the sides of the flowerpot. Finally, (e) changing the plant's posture was done by attaching nylon threads to differents twigs of the plant and pulling at them with a servo, fastened to the side of the flowerpot.

In the second phase, we integrated all functionality into a single Arduino and built a wooden casing to implement all modalities in one place. The water tank was put in a lower compartment of the casing, below the plant itself. In addition to the low-level API implemented on the Arduino, we added high-level functionality on a Raspberry Pi.

\section{DEMONSTRATORS}

In order to prove the feasibility of the overall design, we have developed two demonstrators, which use the different modalities of the InfoPlant:

\section{A. Eco-Feedback}

A central focus of our work lies in the area of sustainable HCI and we are interested in the question, whether more natural interfaces indeed lead to a higher efficacy in terms of behavior change. In order to study the effect of the InfoPlant as an unobtrusive eco-feedback display, we have designed a demonstrator that indicates the current and long-term consumption of 1) electricity, 2) heating energy, and 3) water. For that purpose, we divided the InfoPlant into three sections, each corresponding to one type of consumption and each with its own color: white for electricity, red for heating energy and blue for water. The colors are displayed by individual segments of the LED ring and can be queried by touching the plant, in order to give the user an overview about which part of the plant corresponds to which type of consumption. For all three of these types, the InfoPlant indicates the following information:

1) Current consumption is displayed through the posture of the plant: Three independent leafs (or branches) are being pulled down depending on the current amount (and type) of consumption, resulting in a rather intuitive and potentially emotion-evoking display of this variable.

2) Long-term consumption: As discussed in Section III-1, influencing the overall state of the plant is a comparably slow process, which is why we decided to display long-term consumption with the help of this modality. This could either be based on one (e.g. for the user most important) resource 
type or on a combined value for all of them. Even more than with the indicator for the current consumption, we are aiming for an "emotional" display here.

3) Excessive consumption: Based on thresholds that are determined by analyzing previous consumption patterns, the InfoPlant will inform the user about expenditure that is unusually high: When the current consumption of one type exceeds such a threshold, the respective part of the LED ring starts to glow in its corresponding color. Furthermore, exceeding a second threshhold that indicates a truly excessive consumption will enable the fans to rustle the plant's leafs and let the respective LEDs blink.

\section{B. Display of Communication}

Our second demonstrator is designed to be an unobtrusive alternative to conventional means of notifying the user about communication events, like incoming messages. This allows users to fully concentrate on their current primary activity, while, for example, still being able to keep track of their inbox on an subconscious level. We restricted this demonstrator to notifying the user about events coming from Twitter and e-mail, although integrating more communication services would certainly be possible. By connecting to a user-selected e-mail account through python's imaplib, we can keep track of how many e-mails are in the inbox and get notified, when new messages are coming in. Additionally, we can monitor activites within the user's Twitter account, using tweepy ${ }^{3}$. Mapping this information to the modalities of the InfoPlant, every time a new tweet is posted on the user's wall, we let the leafs rustle for a short amount of time and simultaneously light up the ring of LEDs to display a blue color to indicate Twitterrelated activities. Similarly, when there is incoming e-mail, a short leaf-rustling is triggered and accompanied by the LEDs lighting up with a white color. Additionally, we change the posture of the plant depending on the amount of e-mails that are in the inbox: The more e-mails are waiting to be read, the more will the leafs of the plant be pulled down ${ }^{4}$.

\section{STUDY}

To evaluate both the InfoPlant itself as well as its use as an eco-feedback device, we conducted a small study with the prototype described in Section V-A with $n=15$ participants.

\section{A. Experiment Design}

The study was conducted in a lab in our university that is furnished as a living room with a maximum of four people invited for each trial. A single run lasted 30 minutes and the participants were asked to bring their own work or other activities, which they should concentrate on during the experimental phase. This way, the setting was most similar to the typical scenario where the InfoPlant would be installed at home and the inhabitants would attend to it only passively. In order to keep the different runs comparable and reproducible, instead of manually changing consumption values for the InfoPlant to react to these changes, we scripted a simulated consumption-scenario, which was executed for each trial. After an explanation of how the InfoPlant is displaying the

\footnotetext{
${ }^{3}$ http://www.tweepy.org/, last accessed: 2015-07-02

${ }^{4} \mathrm{~A}$ demo video can be found here: http://tiny.cc/InfoPlant-communication
}

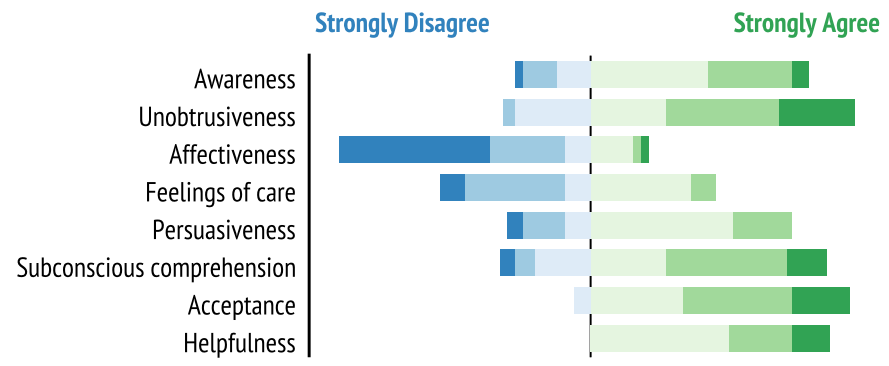

Fig. 4. Condensed results of the questionnaire. Each item corresponds to a number of underlying questions. The answers were given on a 7-point Likerttype scale. Displayed are only the responses that were not "neutral".

consumption information, the only task the participants were given (besides keeping themselves busy with their work) was to $\log$ the current (simulated) consumption in an interactive web applet on an 8-point scale. Each run was recorded on video in order to analyze the reactions of the participants and the interaction with the InfoPlant. Before and after the trial, the participants answered a questionnaire where answers were given on a 7-point Likert-type scale: first on general information about themselves, e.g. if they are easily bothered by background noises or movements, and afterwards specifically about the interaction with the InfoPlant. While presented in a random order, the questions were grouped together to a number of summative Likert scales dealing with specific characteristics (e.g. unobtrusiveness) of the ambient display.

\section{B. Results}

1) Novelty effect: Although the participants have explicitly been given the assignment to keep themselves busy with their work, we could observe that by some of them the InfoPlant was given more direct attention than would seem normal for an ambient information display. The main reason for this we see in the fact that for all participants the plant was a new and interesting object, which naturally made them focus on it more than on their brought work (which they probably knew quite well already) and we can expect it to receive less (direct) attention when people are accustomed to it.

2) Questionnaire results: In Figure 4 we can see the condensed results of the InfoPlant questionnaire. As described in the previous section, each item corresponds to several underlying questions in order to give a more precise picture of the participant's responses. We can see that the InfoPlant was generally well-received and the large majority of people could imagine using it for a longer period of time in their homes (Acceptance). Also it was perceived as rather unobtrusive, e.g. most participants didn't feel distracted by it in their work. In the context of eco-feedback, the InfoPlant was assessed as potentially very helpful and that it would make users aware of their resource consumption. Even after only 30 minutes of exposure, the majority of participants could assert that they would be able to perceive the presented information without conscious effort (Subconscious comprehension). Also, most people responded that this ambient information display could have an influence on their consumption behavior (Persuasiveness).

The most surprising result to us was that only a small percentage of participants reported an emotional reaction to the 
InfoPlant (Affectiveness and Feelings of care). While it indeed might be the case that the ambient display was perceived rather "neutral", we currently assume that the corresponding questions (e.g. "Does the InfoPlant cause an emotional reaction?") were too direct and asked for too extreme emotional reactions for them to capture the probably rather subtle emotional reaction to something as ordinary as a plant.

3) Individual comments: The participants were also given some open-ended questions, e.g. what (if anything) they would potentially find obtrusive or general comments about the InfoPlant. Answers to the first question mainly involved potential distraction caused by any noises that were produced, e.g. from the fans to rustle the leafs or from the servo motor to change the plant's posture. This poses the question if it might be possible to augment these events with an additionally produced sound to make them appear less obtrusive. General comments included a lack in precision for this information display and that more detailed values should be provided additionally. One participant also mentioned that the parallel display of several consumption values might be confusing for the user.

4) Precision of (reported) estimation of consumption: Partly, this could also be seen when comparing the estimation as reported by the participants with the displayed consumption data: A few users occasionally confused two types of consumption, e.g. were indicating an increase in water consumption while instead an increase in heating energy was displayed. Also, while a change in consumption was usually realized, most users could not always precisely determine the exact simulated value.

\section{DISCUSSION AND CONCLUSION}

The results of the study in summary give evidence that a plant can become an unobtrusive ambient information system in smart living rooms or offices and as such an inter-cognitive infocommunication device [23] that enables the user to subonsciously perceive information that otherweise might not be easily accessible. The different modalities are not fully independent, though, as for instance posture control interferes with the overall state, or fan noises could be taken as intentional sounds coming from the plant. Nonetheless, we believe that augmenting the plant artifact with additional information such as energy consumption will be effective in raising awareness to these phenomena, and the questionnaires do support this evaluation. Our experience with users guides us towards a set of plants (e.g. one for every energy type) so that we reduce the risk of misinterpretation and gain the overall-state control for the individual plants. Furthermore, the plant containers could then be designed (e.g. equipped with symbols) that facilitate their association to resources. Of course, more research is necessary, and particularly evaluation in longitudinal studies, to assess quantitative effects on habits and attitudes.

In summary, the InfoPlant demonstrates a novel system design approach for ambient information systems that considers emotional undertones, limited attention, and targets peripheral awareness.

\section{ACKNOWLEDGMENTS}

This research was supported by the Cluster of Excellence Cognitive Interaction Technology 'CITEC' (EXC 277) at
Bielefeld University, which is funded by the German Research Foundation (DFG).

\section{REFERENCES}

[1] M. Weiser, "The Computer for the 21st Century," Scientific American, vol. 265, no. 3, pp. 94-104, Sep. 1991

[2] M. Weiser and J. Brown, "The coming age of calm technology [1]," Xerox PARC. Retrieved July, vol. 8, p. 2007, 1996.

[3] Z. Pousman and J. Stasko, "A taxonomy of ambient information systems," in Proceedings of the working conference on Advanced visual interfaces - AVI '06. New York, New York, USA: ACM Press, May 2006, p. 67.

[4] E. Liscum, "Phototropism: mechanisms and outcomes," The Arabidopsis Book, vol. 51, pp. 1-21, 2002.

[5] D. Holstius, J. Kembel, and A. Hurst, "Infotropism: living and robotic plants as interactive displays," Proceedings of the 5th conference on Designing interactive systems: processes, practices, methods, and techniques (2004), pp. 215-221, 2004.

[6] J. Froehlich, L. Findlater, and J. Landay, "The design of eco-feedback technology," in Proceedings of the 28th international conference on Human factors in computing systems - CHI '10. New York, New York, USA: ACM Press, Apr. 2010, p. 1999.

[7] B. J. Fogg, "Persuasive technology: using computers to change what we think and do," Ubiquity, vol. 2002, no. December, p. 5, 2002.

[8] H. Tanaka and S. Kuribayashi, "Botanical interface design -creative kits, tools, and methods-," pp. 577-584, 2007.

[9] S. Kuribayashi, Y. Sakamoto, and H. Tanaka, "I/O plant," in $C H I$ '07 extended abstracts on Human factors in computing systems - CHI '07. New York, New York, USA: ACM Press, Apr. 2007, p. 2537.

[10] S. Kuribayashi, Y. Sakamoto, M. Morihara, and H. Tanaka, "Plantio," in Proceedings of the international conference on Advances in computer entertainment technology - ACE '07. New York, New York, USA: ACM Press, Jun. 2007, p. 139.

[11] N. D. Christos Goumopoulos, Eleni Christopoulou and A. Kameas, The PLANTS System: Enabling Mixed Societies of Communicating Plants and Artefacts, ser. Lecture Notes in Computer Science, P. Markopoulos, B. Eggen, E. Aarts, and J. L. Crowley, Eds., 2004, vol. 3295.

[12] M. Sato, I. Poupyrev, and C. Harrison, "Touché: enhancing touch interaction on humans, screens, liquids, and everyday objects," Proceedings of the SIGCHI Conference on Human Factors in Computing Systems (CHI '12), pp. 483-492, 2012.

[13] I. Poupyrev, P. Schoessler, J. Loh, and M. Sato, "Botanicus Interacticus: interactive plants technology," SIGGRAPH '12 ACM SIGGRAPH 2012 Emerging Technologies, 2012.

[14] S. Antifakos and B. Schiele, "LaughingLily: Using a flower as a realworld information display," International Conference on Ubiquitous Computing (Ubicomp), 2003.

[15] T. Matthews, T. Rattenbury, and S. Carter, "Defining, designing, and evaluating peripheral displays: An analysis using activity theory," Human-Computer Interaction, vol. 22, no. 1-2, pp. 221-261, 2007.

[16] A. C. Leopold et al., "Plant growth and development." Plant growth and development., 1964.

[17] G. D. Massa, H.-H. Kim, R. M. Wheeler, and C. A. Mitchell, "Plant productivity in response to led lighting," HortScience, vol. 43, no. 7, pp. 1951-1956, 2008.

[18] T. Hermann, A. Hunt, and J. G. Neuhoff, The sonification handbook. Logos Verlag Berlin, GE, 2011.

[19] R. Tünnermann, J. Hammerschmidt, and T. Hermann, "Blended Sonification: Sonification for Casual Interaction," in The 19th International Conference on Auditory Display (ICAD-2013), 2013.

[20] S. Zehe, "BRIX $X_{2}$ : The Xtensible physical computing platform," http: //tiny.cc/brix2.

[21] "VH400 Soil Moisture Sensor Probe," http://vegetronix.com/Products/ $\mathrm{VH} 400 /$.

[22] "Touche for Arduino: Advanced touch sensing," http://www. instructables.com/id/Touche-for-Arduino-Advanced-touch-sensing/. 
[23] P. Baranyi and A. Csapo, "Definition and synergies of cognitive infocommunications," Acta Polytechnica Hungarica, vol. 9, no. 1, pp. $67-83,2012$. 